\title{
The $\mathrm{Ge}(001)(2 \times 1)$ reconstruction: asymmetric dimers and multilayer relaxation observed by grazing incidence $\mathrm{X}$-ray diffraction
}

\author{
R. Rossmann, H.L. Meyerheim, V. Jahns, J. Wever, W. Moritz, D. Wolf, D. Dornisch \\ and $\mathrm{H}$. Schulz \\ Institut für Kristallographie und Mineralogie der Universität München, Theresienstrasse 41, D-8000 München 2, Germany
}

Received 25 June 1992; accepted for publication 25 August 1992

\begin{abstract}
Grazing incidence X-ray diffraction has been used to analyze in detail the atomic structure of the $(2 \times 1)$ reconstruction of the $\mathrm{Ge}(001)$ surface involving far reaching subsurface relaxations. Two kinds of disorder models, a statistical and a dynamical were taken into account for the data analysis, both indicating substantial disorder along the surface normal. This can only be correlated to asymmetric dimers.

Considering a statistical disorder model assuming randomly oriented dimers the analysis of 13 symmetrically independent in-plane fractional order reflections and of four fractional order reciprocal lattice rods up to the maximum attainable momentum transfer $q_{z}=3 c^{*}\left(c^{*}=1.77 \times 10^{-1} \AA^{-1}\right)$ indicates the formation of asymmetric dimers characterized by a dimer bondlength of $R_{\mathrm{D}}=2.46(5) \AA$ as compared to the bulk bonding length of $R=2.45 \AA$. The dimer height of $\Delta z=0.74(15) \AA$ corresponds to a dimer buckling angle of $17(4)^{\circ}$. The data refinement using anisotropic thermal parameters leads to a bonding length of $R_{\mathrm{D}}=2.44(4) \AA$ and to a large anisotropy of the root mean-square vibration amplitudes of the dimer atoms $\left(\left\langle u_{11}^{2}\right\rangle\right)^{1 / 2}=0.25 \AA$, $\left.\left(\left\langle u_{22}^{2}\right\rangle\right)^{1 / 2}=0.14 \AA,\left(\left\langle u_{33}^{2}\right\rangle\right)^{1 / 2}=0.50 \AA\right)$. We have evidence for lateral and vertical displacements of the Ge atoms down to the tenth layer below the surface.
\end{abstract}

\section{Introduction}

The geometric and electronic structure of the $(2 \times 1)$ reconstruction of the (001) surfaces of germanium and silicon have been of considerable interest in recent years [1-12]. Although there is no dispute about the formation of dimers, the question whether the dimers are symmetric or asymmetric is still a matter of dispute. Scanning tunneling microscopy (STM) spectroscopy [9] and recent density functional calculations [13] for $\mathrm{Si}(100)(2 \times 1)$ favor the formation of asymmetric dimers, where a dynamical flipping $\left(\nu=10^{9} \mathrm{~Hz}\right)$ of the dimers is proposed to explain the observation of symmetric dimers in STM topographs [5]. The asymmetry of the dimers has now been confirmed by temperature dependent STM analyses of Wolkow [14] for the Si(100) surface.

Contrary to silicon, the STM investigation of the $\mathrm{Ge}(001)(2 \times 1)$ reconstruction [7] shows the presence of asymmetric dimers at room temperature which do not require vacancy type defects as supposed for $\operatorname{Si}(10)(2 \times 1)$. Furthermore, recent transmission electron microscopy (TEM) [11] and angular resolved photoemission (ARUPS) data compared with calculations [10] support the picture of asymmetric dimers. This is also confirmed by energy minimization calculations by Needels et al. [8] as well as by $\mathrm{He}^{+}$ion scattering experiments of Culbertson et al. [6], both suggesting a dimer buckling of $14^{\circ}$ and $\sim 20^{\circ}$, respectively.

Previous surface X-ray diffraction measurements [15-17] could not distinguish between symmetric and asymmetric dimers since the resolution normal to the sample surface was not sufficient. Nevertheless, from the data analysis of fractional order in plane intensities of Si(100) $(2 \times 1)$, Jedrecy et al. [18] indirectly concluded that asymmetric dimers are formed which are inclined by $7.4^{\circ}$ to the surface plane. 
A direct analysis of the reconstructed surface is only possible by measuring the fractional order intensities up to high momentum transfers $q_{z}=c^{*}$ normal to the sample surface. Therefore we use a new type of diffractometer allowing both, in-plane and out-of-plane measurements [19].

\section{Measurements}

Prior to mounting into the UHV chamber the $\operatorname{Ge}(001)$ crystal $(15 \mathrm{~mm} \times 10 \mathrm{~mm} \times 1 \mathrm{~mm})$ was polished chemomechanically by diamond paste and a $12 \% \mathrm{NaClO}$ solution. The crystal cleaning was performed by repetitive cycles of $\mathrm{Ar}^{+}$ion sputtering $(500 \mathrm{eV})$ and annealing to $650^{\circ} \mathrm{C}$ by direct heating. After annealing the sample was cooled down to room temperature within $\sim 30$ min. A clear $(2 \times 1)$ LEED pattern with no evidence for the formation of the $\mathrm{c}(2 \times 4)$ or $\mathrm{p}(2 \times 2)$ structure could be observed after this procedure. The X-ray diffraction measurements were performed using $\mathrm{Cu} \mathrm{K} \alpha$ radiation provided by a 18 $\mathrm{kW}$ rotating anode generator monochromatized by a pyrolytic graphite monochromator.

The data collection was also carried out using synchrotron radiation at the beamline $\mathrm{W}_{1}$ of the Hamburger Synchrotonstrahlungslabor (HASYLAB). Both data sets are in agreement with each other, however, the results presented in this paper are from the rotating anode experiments only because the quality of the sample was better.

In order to enhance the signal-to-background ratio the diffraction experiments were performed at an incidence angle of $\alpha_{\mathrm{i}}=0.32^{\circ}$ corresponding to the critical angle of total external reflection. The alignment of the sample was carefully checked by monitoring the intensity and angular position of the totally reflected beam relative to the primary beam using a position sensitive detector. The measurements were performed at room temperature by rotating the crystal about the surface normal ( $\omega$-scan).

Fig. 1 shows an $\omega$-scan over the $(3 / 21)$ reflection with a Lorentzian fit through the data points assuming a linear background shown as dashed line. The width of the reflection curve gives an estimation of the average terrace size of $\sim 200$

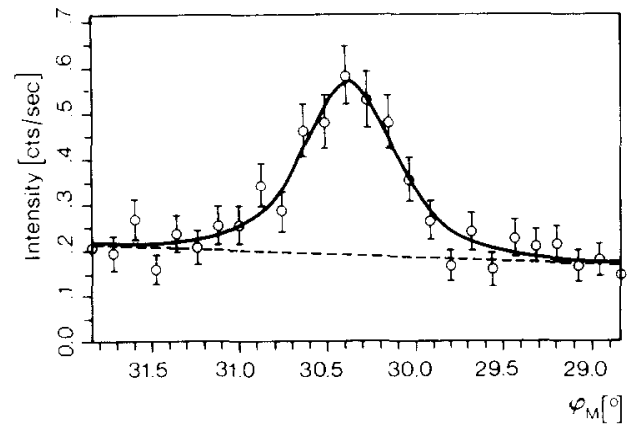

Fig. 1. $\omega$-scan of the $(3 / 21)$ in-plane superlattice reflection. A Lorentzian fit and a linear background are shown as solid and dashed lines, respectively. The reflections are indexed on the basis of the primitive setting of the unreconstructed surface unit cell.

$\AA$, much less than an average of $550 \AA$ given by the sample miscut of $0.15^{\circ}$.

Using Soller-slits an in-plane resolution of $\Delta \phi$ $=0.4^{\circ}$ was achieved corresponding to $\Delta q_{\|}=4.5$ $\times 10^{-3} \AA^{-1}$, by which the background could be sufficiently reduced. In the out-of-plane direction the angular resolution was $\Delta \alpha_{\mathrm{f}}=2.2^{\circ}$ providing a resolution of $\Delta q_{z}=2.5 \times 10^{-2} \AA^{-1}$ corresponding to $0.14 c^{*}$, where $c^{*}=1 / c_{0}=(5.658 \AA)^{-1}=$ $0.177 \AA^{-1}$ is the reciprocal lattice unit normal to the sample surface. It should be noted that for the out-of-plane measurements these values change considerably depending on the detector position. This has to be taken into account by a modified Lorentz factor which was calculated here by numerical integration including the divergences of the primary beam and assuming Lorentzian beam profiles [20]. An analytic solution assuming Gaussian profiles has been described in ref. [21]. In total 42 in-plane reflections were measured which are reduced to 13 by symmetry equivalence. Four superstructure rods, $(1 / 2$ $0),(3 / 20),(3 / 21)$ and $(1 / 21)$, were measured in steps of $\Delta l=0.1-0.2$. Generally, the reproducibility of symmetry equivalent intensities was in most cases within $15-25 \%$. The measured intensities are given in table 1. After background subtraction and correcting the mcasured intensities for variation of the active sample area, polarization and Lorentz factor a total of 80 symmetry inequivalent intensities were obtained. The mea- 
sured structure factor intensities (filled circles) of the superstructure rods are shown in fig. 2 versus $l=q_{z} / c^{*}$, the momentum transfer normal to the sample surface. As solid lines we have plotted the calculated structure factor intensities for the best fit model which will be discussed below.

The comparison with the data of Grey et al. [16] who could only measure up to $l=0.8$ gives reasonable agreement. Qualitatively, the observed rapid intensity variation along $q_{z}$ indicates far reaching displacements of the atomic positions normal to the sample surface.

\section{Analysis of the dimer structure}

Starting point of the data analysis was the structure model proposed by Grey et al. [16]. Using both, the in-plane and out-of-plane fractional intensities two structure models were employed to fit the measured data. We used the fractional order intensities only, since the mea- surement of the integer order beams is affected with systematic errors. These arise from surface inhomogeneities influencing the integer order beams and from the low resolution normal to the sample surface preventing the precise measurement of the intensity close to the Bragg-points.

The first model, called in the following the dynamical model, uses anisotropic thermal parameters $B$ for the dimer atoms. For the second and deeper layers the thermal parameters were assumed to be isotropic and were kept constant at $B=0.80 \AA^{2}$ (second layer), at $B=0.60 \AA^{2}$ (third layer) and $B=0.50 \AA^{2}$ (deeper layers), the latter corresponds approximately to the bulk value [22].

As a second model we considered a disordered surface assuming randomly oriented dimers. This model uses isotropic and constant thermal parameters for all layers (first layer: $B=1.30 \AA^{2}$, second layer: $B=0.70 \AA^{2}$, third layer: $B=0.60$ $\AA^{2}$, deeper layers: $B=0.50 \AA^{2}$ ). The atoms in the first and second layer are allowed to split into
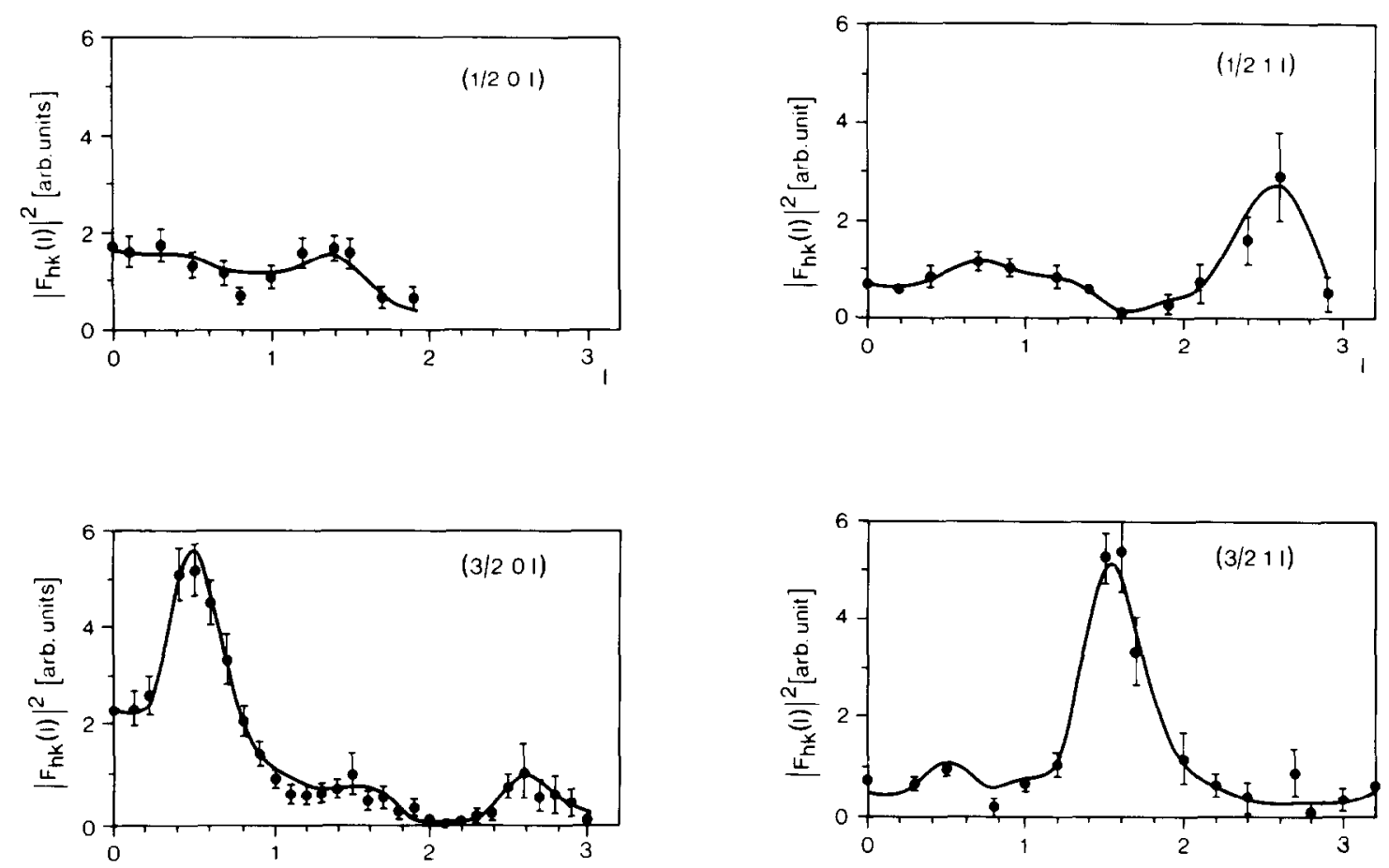

Fig. 2. Measured structure factor intensities of four fractional order superstructure rods versus $l=q_{z} / c^{*}$. The best fit results are shown as solid lines. The rapid intensity oscillations qualitatively indicate far reaching layer relaxations. 
Table 1

Measured intensities for $\mathrm{Ge}(001)(2 \times 1)$ after correcting for active sample area, Lorentz- and polarisation factor

\begin{tabular}{|c|c|c|c|c|}
\hline$h$ & $k$ & 1 & $I$ & $\sigma(I)(\%)$ \\
\hline$\overline{1 / 2}$ & 0 & 0.0 & 251 & 15 \\
\hline $1 / 2$ & 1 & 0.0 & 104 & 5 \\
\hline $3 / 2$ & 0 & 0.0 & 328 & 5 \\
\hline $3 / 2$ & 1 & 0.0 & 107 & 5 \\
\hline $5 / 2$ & 1 & 0.0 & 28 & 50 \\
\hline $1 / 2$ & 3 & 0.0 & 34 & 40 \\
\hline $7 / 2$ & 0 & 0.0 & 32 & 100 \\
\hline $5 / 2$ & 2 & 0.0 & 10 & 40 \\
\hline $3 / 2$ & 3 & 0.0 & 36 & 100 \\
\hline $7 / 2$ & 1 & 0.0 & 108 & 10 \\
\hline $1 / 2$ & 4 & 0.0 & 36 & 150 \\
\hline $5 / 2$ & 3 & 0.0 & 11 & 100 \\
\hline $7 / 2$ & 2 & 0.0 & 37 & 100 \\
\hline $1 / 2$ & 0 & 0.1 & 233 & 20 \\
\hline $1 / 2$ & 0 & 0.3 & 250 & 20 \\
\hline $1 / 2$ & 0 & 0.5 & 189 & 20 \\
\hline $1 / 2$ & 0 & 0.7 & 165 & 20 \\
\hline $1 / 2$ & 0 & 0.8 & 97 & 20 \\
\hline $1 / 2$ & 0 & 1.0 & 153 & 20 \\
\hline $1 / 2$ & 0 & 1.2 & 226 & 20 \\
\hline $1 / 2$ & 0 & 1.4 & 243 & 15 \\
\hline $1 / 2$ & 0 & 1.5 & 226 & 20 \\
\hline $1 / 2$ & 0 & 1.7 & 93 & 30 \\
\hline $1 / 2$ & 0 & 1.9 & 92 & 30 \\
\hline $1 / 2$ & I & 0.2 & 84 & 15 \\
\hline $1 / 2$ & 1 & 0.4 & 124 & 20 \\
\hline $1 / 2$ & 1 & 0.7 & 168 & 15 \\
\hline $1 / 2$ & 1 & 0.9 & 149 & 15 \\
\hline $1 / 2$ & I & 1.2 & 121 & 25 \\
\hline $1 / 2$ & 1 & 1.4 & 84 & 50 \\
\hline $1 / 2$ & 1 & 1.6 & 16 & 80 \\
\hline $1 / 2$ & 1 & 1.9 & 40 & 60 \\
\hline $1 / 2$ & 1 & 2.1 & 101 & 50 \\
\hline $1 / 2$ & 1 & 2.4 & 233 & 30 \\
\hline $1 / 2$ & 1 & 2.6 & 422 & 30 \\
\hline $1 / 2$ & 1 & 2.9 & 74 & 70 \\
\hline $3 / 2$ & 0 & 0.1 & 339 & 15 \\
\hline $3 / 2$ & 0 & 0.2 & 377 & 15 \\
\hline $3 / 2$ & 0 & 0.4 & 736 & 10 \\
\hline $3 / 2$ & 0 & 0.5 & 749 & 10 \\
\hline $3 / 2$ & 0 & 0.6 & 651 & 10 \\
\hline $3 / 2$ & 0 & 0.7 & 483 & 15 \\
\hline $3 / 2$ & 0 & 0.8 & 297 & 10 \\
\hline $3 / 2$ & 0 & 0.9 & 209 & 15 \\
\hline $3 / 2$ & 0 & 1.0 & 137 & 20 \\
\hline $3 / 2$ & 0 & 1.1 & 88 & 30 \\
\hline $3 / 2$ & 0 & 1.2 & 87 & 30 \\
\hline $3 / 2$ & 0 & 1.3 & 91 & 30 \\
\hline $3 / 2$ & 0 & 1.4 & 105 & 25 \\
\hline
\end{tabular}

Table 1 (continued)

\begin{tabular}{|c|c|c|c|c|}
\hline$h$ & $k$ & $l$ & $I$ & $\sigma(I)(\%)$ \\
\hline $3 / 2$ & 0 & 1.5 & 150 & 40 \\
\hline $3 / 2$ & 0 & 1.6 & 70 & 35 \\
\hline $3 / 2$ & 0 & 1.7 & 80 & 40 \\
\hline $3 / 2$ & 0 & 1.8 & 38 & 50 \\
\hline $3 / 2$ & 0 & 1.9 & 51 & 40 \\
\hline $3 / 2$ & 0 & 2.0 & 13 & 100 \\
\hline $3 / 2$ & 0 & 2.1 & 1 & 500 \\
\hline $3 / 2$ & 0 & 2.2 & 9 & 300 \\
\hline $3 / 2$ & 0 & 2.3 & 26 & 60 \\
\hline $3 / 2$ & 0 & 2.4 & 35 & 40 \\
\hline $3 / 2$ & 0 & 2.5 & 114 & 30 \\
\hline $3 / 2$ & 0 & 2.6 & 159 & 50 \\
\hline $3 / 2$ & 0 & 2.7 & 86 & 50 \\
\hline $3 / 2$ & 0 & 2.8 & 91 & 60 \\
\hline $3 / 2$ & 0 & 2.9 & 67 & 60 \\
\hline $3 / 2$ & 0 & 3.0 & 23 & 200 \\
\hline $3 / 2$ & 1 & 0.3 & 97 & 15 \\
\hline $3 / 2$ & 1 & 0.5 & 144 & 15 \\
\hline $3 / 2$ & 1 & 0.8 & 25 & 80 \\
\hline $3 / 2$ & 1 & 1.0 & 98 & 15 \\
\hline $3 / 2$ & l & 1.2 & 155 & 20 \\
\hline $3 / 2$ & 1 & 1.5 & 762 & 10 \\
\hline $3 / 2$ & 1 & 1.6 & 780 & 15 \\
\hline $3 / 2$ & 1 & 1.7 & 486 & 20 \\
\hline $3 / 2$ & 1 & 2.0 & 174 & 40 \\
\hline $3 / 2$ & 1 & 2.2 & 94 & 35 \\
\hline $3 / 2$ & 1 & 2.4 & 62 & 100 \\
\hline $3 / 2$ & 1 & 2.7 & 134 & 50 \\
\hline $3 / 2$ & 1 & 2.8 & 13 & 300 \\
\hline $3 / 2$ & 1 & 3.0 & 49 & 70 \\
\hline $3 / 2$ & 1 & 3.2 & 46 & 80 \\
\hline
\end{tabular}

The reflections are indexed on the basis of the primitive setting of the (unreconstructed) surface unit cell.

two different positions, where each atom is given a statistical weight of $1 / 2$. This model simulates a statistical (coherent) averaging over both dimer orientations within one domain as shown in fig. 3 .

Both models basically lead to the same structure parameters. The best refinement for the dynamical and for the disorder model are summarized in tables 2 and 3 . We have listed the atomic positions in relative coordinate $\left(a_{0}=8.000\right.$ $\AA, b_{0}=4.000 \AA, c_{0}=5.658 \AA$ ) and the absolute displacements of the Ge-atoms from their bulk positions. For the structure refinements we obtained residuals in the $13-14 \%$ range which did not depend on whether we refined the data on weighted $|F|$ or whether we used unit weights. 
Table 2

Structure parameters of the $\operatorname{Ge}(001)(2 \times 1)$ reconstruction on the basis of the dynamical model

\begin{tabular}{|c|c|c|c|c|c|c|}
\hline Atom & $X$ & $Y$ & $Z$ & $B\left(\AA^{2}\right)$ & $\Delta X(\AA)$ & $\Delta Z(\AA)$ \\
\hline 1 & $0.347(3)$ & 0.5 & $0.06(7)$ & & $+0.78(1)$ & $+0.34(40)$ \\
\hline$B_{11}$ & & & & 4.9 & & \\
\hline$B_{22}$ & & & & 1.6 & & \\
\hline$B_{33}$ & & & & 19.7 & & \\
\hline 2 & $0.262(1)$ & 0.0 & $0.23(7)$ & 0.8 & $0.10(1)$ & $-0.1(4)$ \\
\hline $3_{1}$ & $0.000^{*}$ & 0.0 & $0.49(7)$ & 0.6 & $0.00^{*}$ & $-0.1(4)$ \\
\hline $3_{2}$ & $0.500^{*}$ & 0.0 & $0.55(7)$ & 0.6 & $0.00 *$ & $+0.3(4)$ \\
\hline $4_{1}$ & $0.000^{*}$ & 0.5 & $0.77(7)$ & 0.5 & $0.00^{*}$ & $+0.1(4)$ \\
\hline $4_{2}$ & $0.500^{*}$ & 0.5 & $0.81(7)$ & 0.5 & $0.00 *$ & $-0.3(4)$ \\
\hline $5^{2}$ & 0.243 (1) & 0.5 & $1.02(7)$ & 0.5 & $-0.06(1)$ & $+0.1(4)$ \\
\hline 6 & $0.246(1)$ & 0.0 & $1.18(7)$ & 0.5 & $-0.03(1)$ & $-0.4(4)$ \\
\hline $7_{1}$ & $0.000^{*}$ & 0.0 & $1.53(7)$ & 0.5 & $0.00^{*}$ & $+0.2(4)$ \\
\hline 72 & $0.500^{*}$ & 0.0 & $1.54(7)$ & 0.5 & $0.00 *$ & $+0.2(4)$ \\
\hline $8_{1}$ & $0.000^{*}$ & 0.5 & $1.78(9)$ & 0.5 & $0.00 *$ & $+0.2(5)$ \\
\hline 82 & $0.500^{*}$ & 0.5 & $1.79(9)$ & 0.5 & $0.00 *$ & $+0.2(5)$ \\
\hline 9 & $0.257(2)$ & 0.5 & $1.96(7)$ & 0.5 & $+0.06(2)$ & $-0.2(4)$ \\
\hline 10 & $0.254(2)$ & 0.0 & 2.25 & 0.5 & $+0.03(2)$ & 0.0 \\
\hline
\end{tabular}

The atoms are numbered according to their sequence in the structure beginning with 1 (top layer, see fig. 5). The derived lateral and vertical $(\Delta X, \Delta Z)$ displacements of the Ge-atoms from the bulk lattice positions are given in Angströms. The parameters labeled by * are fixed due to symmetry.

In all cases the goodness of fit parameter (GOF) was close to one indicating that the calculated structure factors agree within the error bars with the experimental data [17].
For the disorder model the Fourier map (fig. 4) is shown for two sections $y=0$ (left) and $y=1 / 2$ (right). The filled circles indicate the bulk atomic positions of the germanium atoms,

Table 3

Structure parameters of the $\mathrm{Ge}(001)(2 \times 1)$ reconstruction on the basis of the statistical model

\begin{tabular}{cllllll}
\hline Atom & $X$ & $Y$ & $Z$ & $B\left(\AA^{2}\right)$ & $\Delta X(\AA)$ & $\Delta Z(\AA)$ \\
\hline $1 \mathrm{a}$ & $0.327(8)$ & 0.5 & $-0.06(7)$ & 1.3 & $+0.62(7)$ & $-0.3(1)$ \\
$1 \mathrm{~b}$ & $0.379(4)$ & 0.5 & $0.07(2)$ & 1.3 & $+1.03(3)$ & $+0.4(1)$ \\
$2 \mathrm{a}$ & $0.292(8)$ & 0.0 & $0.22(2)$ & 0.7 & $+0.34(7)$ & $-0.2(1)$ \\
$2 \mathrm{~b}$ & $0.243(6)$ & 0.0 & $0.29(9)$ & 0.7 & $-0.06(5)$ & $+0.2(5)$ \\
$3_{1}$ & $0.000^{*}$ & 0.0 & $0.44(2)$ & 0.6 & $0.00^{*}$ & $-0.3(1)$ \\
$3_{2}$ & $0.500^{*}$ & 0.0 & $0.49(2)$ & 0.6 & $0.00^{*}$ & $-0.1(1)$ \\
$4_{1}$ & $0.000^{*}$ & 0.5 & $0.71(2)$ & 0.5 & $0.00^{*}$ & $-0.2(1)$ \\
$4_{2}$ & $0.500^{*}$ & 0.5 & $0.74(2)$ & 0.5 & $-0.07(1)$ & $-0.1(1)$ \\
5 & $0.241(1)$ & 0.5 & $0.98(4)$ & 0.5 & $-0.03(1)$ & $-0.1(2)$ \\
6 & $0.246(1)$ & 0.0 & $1.21(4)$ & 0.5 & $0.00^{*}$ & $-0.2(2)$ \\
$7_{1}$ & $0.000^{*}$ & 0.0 & $1.43(4)$ & 0.5 & $0.00^{*}$ & $-0.4(2)$ \\
$7_{2}$ & $0.500^{*}$ & 0.0 & $1.44(4)$ & 0.5 & $0.00^{*}$ & $-0.3(2)$ \\
$8_{1}$ & $0.000^{*}$ & 0.5 & $1.69(3)$ & 0.5 & $0.00^{*}$ & $-0.3(2)$ \\
$8_{2}$ & $0.500^{*}$ & 0.5 & $1.70(3)$ & 0.5 & $+0.06(3)$ & $-0.3(2)$ \\
9 & $0.257(4)$ & 0.5 & $1.93(4)$ & 0.5 & $+0.02(3)$ & $0.4(2)$ \\
10 & $0.253(4)$ & 0.0 & 2.25 & 0.5 & \\
\hline
\end{tabular}

The aloms in layers 1 and 2 are splitted and are labeled by a and $b$, respectively. The atomic coordinates and the shifts are listed as in table 1. 


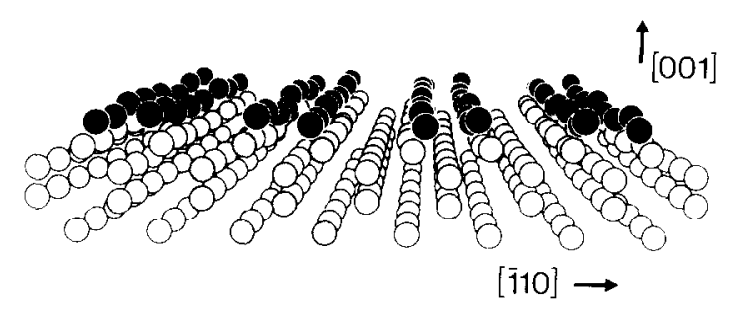

Fig. 3. Schematic view of the disordered dimer model structure involving a statistical orientation of buckled dimers (black circles) within one domain. The buckling of the second layer atoms is also shown on scale. Atoms in layers 2 to 5 represented by open circles are plotted to indicate the bulk repeating distance along the (reconstructed) [110] direction.

wheras the open circles represent the derived atomic positions on the basis of the disorder model.
It can be seen that due to the shifts of the Ge atoms large positive maxima (solid lines) are induced. Since only fractional order structure factors have been used, the Fourier transform provides a picture of the difference structure relative to the $(1 \times 1)$ bulk structure. This gives also rise to the occurrence of negative peaks in the map indicated by the dashed lines. The low number of structure factors distorts the Fourier map due to truncation errors. Nevertheless, the most important features of the superstructure can be seen and to our knowledge this is the first depth resolved Fourier synthesis obtained from a superstructure.

The presence of dimers is directly evident by the intense maxima at $x \approx 0.35, y=1 / 2$ and

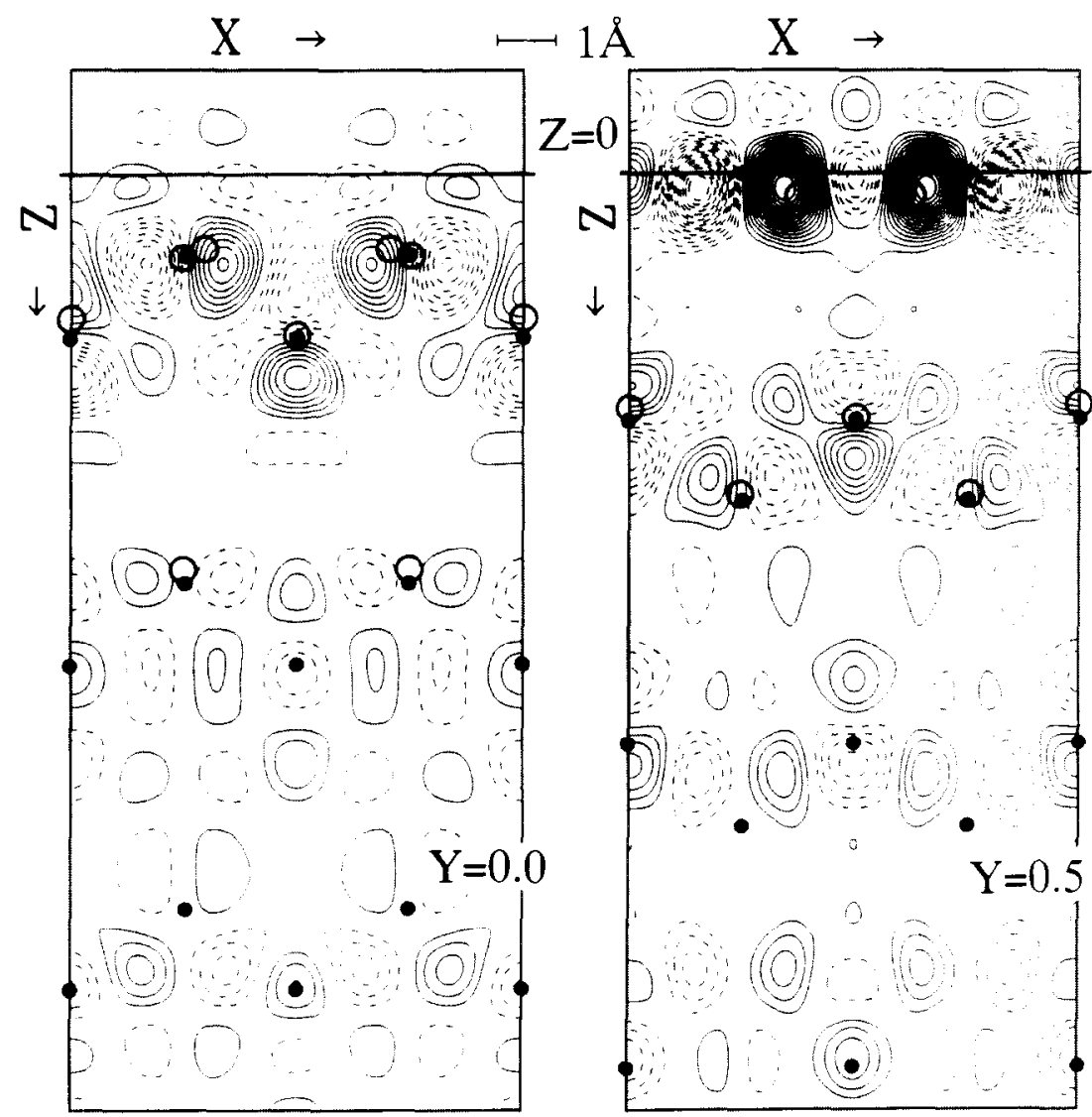

Fig. 4. Fourier map in the $x-z$ plane derived from fractional order structure factors only. Sections $y=0$ (left) and $y=1 / 2$ (right) are shown, the latter containing the dimer atoms. Along the $x$-axis one complete reconstructed unit cell is shown ( $a_{0}=8.000 \AA$ ). The filled circles indicate bulk atomic positions, the open circles show the positions of the Ge atoms derived on the basis of the statistical disorder model. Positive and negative difference maxima are plotted as solid and dashed lines, respectively. 

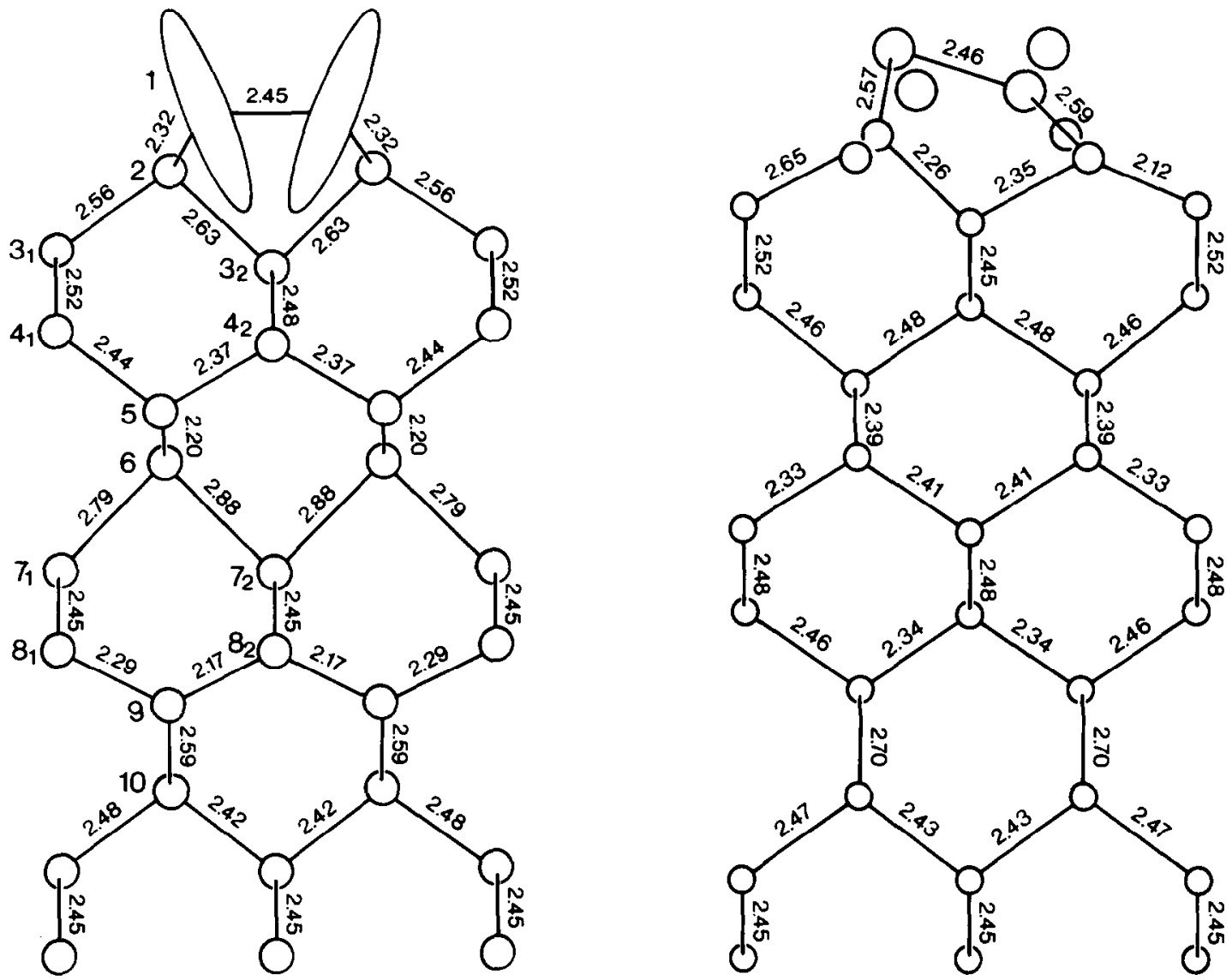

Fig. 5. Derived bond lengths for the dynamical model (left) and the statistical disorder model (right). For the dynamical model the vibration ellipsoid is shown schematically. The atoms are numbered in correspondence with tables 1 and 2 . The bulk bonding length is $R=2.45 \AA$. Note that the error bar associated with the bonding lengths is in the order of $0.6 \AA$ due to the limited data set.

$z=0$. Note that this maximum appears very elongated along the $z$-direction as compared to other maxima. This qualitatively indicates a large disorder along $z$.

An average dimer bond length of $R_{\mathrm{D}}=2.44(4)$ $\AA$ is obtained using the dynamical model. This is close to the bulk value of $2.45 \AA$. We derived anisotropic thermal parameters of $B_{11}=4.93 \AA^{2}$, $B_{22}=1.55 \AA^{2}$ and $B_{33}=19.7 \AA^{2}$, corresponding to root mean-square (rms) displacements of $\left(\left\langle u_{11}^{2}\right\rangle\right)^{1 / 2}=0.25 \AA$, $\left(\left\langle u_{22}^{2}\right\rangle\right)^{1 / 2}=0.14 \AA$ and $\left(\left\langle u_{33}^{2}\right\rangle\right)^{1 / 2}=0.50 \AA$, respectively. The rms displacement of $\left(\left\langle u_{33}^{2}\right\rangle\right)^{1 / 2}=0.50 \AA$ corresponds to a maximum buckling angle of $\alpha=21^{\circ}$.
There is a significant anisotropy of the in-plane vibration amplitudes as well. The thermal disorder is more confined within the $x-z$ plane compared to the $y-z$ plane indicating that twisting of the dimers out of the $x-z$ plane is less important.

These results were obtained keeping the second and deeper layer thermal parameters constant (see above). Due to the limited data set it is not possible to refine all parameters in the structure refinement.

Using the disorder model the splitting of the dimer atoms was derived to be $\Delta z=0.74(15) \AA$ resulting in a dimer bond length of $R_{\mathrm{D}}=2.46(5)$ $\AA$ and to a buckling angle of $17(4)^{\circ}$. The splitting 
$\Delta z$ and the rms displacement $\left(\left\langle u_{33}^{2}\right\rangle\right)^{1 / 2}=0.50 \AA$ determined in the dynamical model are roughly equivalent, both indicating substantial disorder along the $z$-direction which can only be explained by the presence of asymmetric dimers.

The results presented here are in agreement with other experimental and theoretical results for the Ge(001) $(2 \times 1)$ and the $\operatorname{Si}(001)(2 \times 1)$ reconstruction. For the latter the dynamical model appears now to be most convincing [14]. The most prominent features of the dynamical model are very large thermal parameters and their anisotropy. This can be correlated with the flipping model of Dabrovsky and Scheffler [13] who calculated a maximum buckling angle of $\alpha=15^{\circ}$. In contrast, the X-ray data of Jedrecy et al. [18] lead only to $7.4^{\circ}$ for Si. However, this result was obtained indirectly by measuring in-plane data only. On the basis of $\mathrm{He}^{+}$ion-scattering experiments Culbertson et al. [6] derive a buckling angle of $\sim 20^{\circ}$ for $\operatorname{Ge}(001)(2 \times 1)$, whereas Needels et al. [8] calculate a buckling of $14^{\circ}$ leading to an energy gain of $0.24 \mathrm{eV}$ per dimer.

Considering the dimer bond length the dynamical model (derived bond length $R_{\mathrm{D}}=2.45(4) \AA$ ) leads to a larger value as compared to Grey's et al. [16] results of $R_{\mathrm{D}}=2.32(4)$ and $2.36(4) \AA$. In this context it has to be kept in mind that the latter are projected bonding lengths only. There-

Table 4

Comparison between calculated and experimentally derived displacements (statistical disorder model)

\begin{tabular}{lccccc}
\hline Atom & \multicolumn{2}{l}{ Keating model } & & \multicolumn{2}{l}{ Experiment } \\
\cline { 2 - 3 } \cline { 5 - 6 } & $\Delta X(\AA)$ & $\Delta Z(\AA)$ & & $\Delta X(\AA)$ & $\Delta Z(\AA)$ \\
\hline 1 & +0.744 & -0.158 & & $+0.78(1)$ & $+0.3(4)$ \\
2 & +0.104 & -0.002 & & $+0.10(1)$ & $-0.1(4)$ \\
$3_{1}$ & $0.000^{*}$ & -0.136 & & $0.00^{*}$ & $-0.1(4)$ \\
$3_{2}$ & $0.000^{*}$ & +0.136 & & $0.00^{*}$ & $+0.3(4)$ \\
$4_{1}$ & $0.000^{*}$ & -0.096 & & $0.00^{*}$ & $+0.1(4)$ \\
$4_{2}$ & $0.000^{*}$ & +0.096 & & $0.00^{*}$ & $-0.3(4)$ \\
5 & -0.052 & $+0.000^{*}$ & $-0.06(1)$ & $+0.1(4)$ \\
6 & -0.016 & $+0.000^{*}$ & $-0.03(1)$ & $-0.4(4)$ \\
$7_{1}$ & $0.000^{*}$ & +0.016 & & $0.00^{*}$ & $+0.2(4)$ \\
$7_{2}$ & $0.000^{*}$ & -0.011 & & $0.00^{*}$ & $+0.2(4)$ \\
$8_{1}$ & $0.000^{*}$ & +0.011 & & $0.00^{*}$ & $+0.2(5)$ \\
$8_{2}$ & $+0.000^{*}$ & -0.011 & & $0.00^{*}$ & $+0.2(5)$ \\
\hline
\end{tabular}

The calculation [23] is based on the Keating-model and assumes symmetric dimers. fore, by comparing the results of the statistical model we have to average over the positions of the split atoms leading to a projected $R_{\mathrm{D}}=$ 2.35(7) $\AA$ which is in good agreement with Grey's et al. data.

We could not find any evidence for the formation of $\mathrm{p}(2 \times 2)$ and $\mathrm{c}(4 \times 2)$ superstructures, although on the basis of $\mathrm{X}$-ray measurements it is not possible to exclude the formation of small locally ordered patches which were observed by Kubby et al. [7] using STM.

Finally, we want to note that our experimental data cannot provide a distinction between dynamical flipping and randomly disordered asymmetrical dimers. A temperature dependent measurement might rule out the unlikely possibility of a large amplitude simple harmonic vibration of the dimers, because it would diminish at low temperature.

\section{Layer relaxation}

The structure refinement of our data set indicates lateral and vertical displacements several layers deep giving rise to a rapid oscillation of the superstructure rod intensity shown in fig. 2 . The overall agreement between fit and experiment is quite good. Qualitatively, the subsurface relaxations can be observed in the Fourier map (fig. 4) down to the sixth layer, although the corresonding maxima are considerably distorted. Relaxations in deeper layers are less prominent but still significant in the analysis. A schematic view of the structure is shown in fig. 5 for both models. The atoms are numbered corresponding to the tables 2 and 3 . Since there is no other structure analysis available providing atomic positions ten layers deep, we compare in table 4 the derived displacements of the germanium atoms with those obtained by the Keating model for $\operatorname{Ge}(001)(2 \times 1)$ reconstruction [23]. However, this model assumes symmetric dimers and therefore the direct comparison may be questionable. Nevertheless, especially for the lateral displacements where the experimental error bars are small the agreement between experimental and calculated displacements is reasonably well. For the vertical dis- 
placements the error bars are much larger and a comparison appears not to be useful. In fig. 5 the derived bond lengths for both models are indicated. Some of them differ by more than $10 \%$ from the bulk bond length $R=2.45 \AA$ ) which appears to be unphysical. This is especially true for the bonds between the sixth and seventh layer. However, this should not be overestimated since the error bars of the atom displacements are very large. For example, from tables 2 and 3 it can be seen that the error bars associated with the bonding lengths are generally in the regime of $\sim 0.6 \AA$. This uncertainty is a consequence of the limited number of rods that could be measured due to the low flux provided by the rotating anode. Additionally, changing the data analysis procedure from refining on weighted $|F|$ to refinement on unweighted $|F|$ results in changes of the bonding lengths which in most cases is in the order of $0.2-0.3 \AA$, considerably smaller than the total error bar.

Some unrealistic bond lengths are removed by
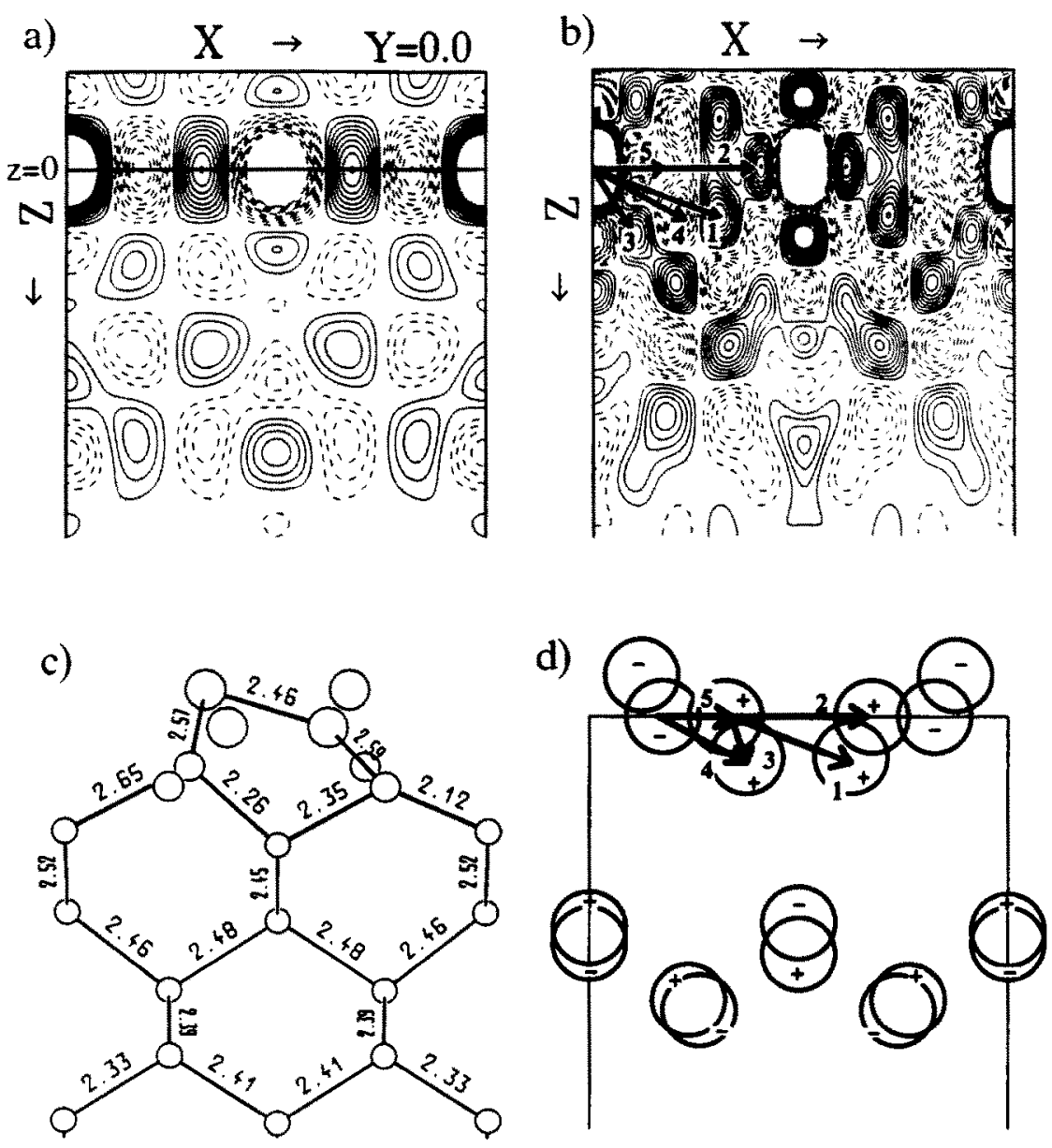

Fig. 6. (a) Patterson-map derived from 80 measured structure factor intensities. Positive and negative maxima are indicated by solid and dashed lines, respectively. (b) Patterson-map including 429 structure factor intensities calculated on the basis of the statistical disorder model. The vectors labeled 1-5 correspond to the difference electron densities shown in. (c) View of the statistical disorder model. (d) Schematic plot of the difference electron density resulting from displacements of the atoms from their bulk $(1 \times 1)$ positions. Only layers 1,4 and 5 are considered. The vectors labeled $1-5$ between the difference electron densities are compared with positive and negative maxima of the Patterson-map shown in (a) and (b). 
including more data into the refinement such as integer order reflections [24]. Nevertheless, we want to note that although the vertical atomic positions are affected with large error bars we have evidence for the reconstruction extending at least to the tenth layer, since by including the ninth and tenth layer into the refinement procedure the GOF dropped from 1.21 to 0.93 , where $\sigma_{\mathrm{GOF}}=0.13$.

\section{Interpretation of the Patterson function}

Using the measured data set of 80 symmetry inequivalent reflections we calculated the Patterson function which is shown in fig. 6a. It includes only those vectors that have no component out of the $x-z$ plane (section $y=0$ ). Additionally, we confine our consideration to the plane which contains the dimer atoms (layers 1, 4 and 5).

Since only superlattice reflections have been used both, positive and negative peaks (dashed lines) are observable. The partial Patterson function is the autocorrelation function of the difference structure relative to the averaged $(1 \times 1)$ structure. The averaged $(1 \times 1)$ structure is constructed by averaging the superstructure over both, the $(1 \times 1)$ sites and the superstructure sites [25].

Due to the shifts of the Ge atoms out of the $(1 \times 1)$ positions "dipoles" of positive and negative difference electron density relative to the averaged $(1 \times 1)$ structure are created (fig. $6 \mathrm{~d})$.

Some vectors, labeled 1-5 connecting the difference densities are shown. Depending on the combination of the signs of the difference densities these should be correlated to positive (combinations:,$++ \quad-)$ ) and negative (combinations: $+-,-+)$ densities in the Patterson function.

By comparison of the vectors 1-5 with the experimental Patterson function (fig. 6a) it becomes evident that there is no straightforward correlation. The most important reason is the limited number of measured Fourier-components. For comparison we show in fig. $6 \mathrm{~b}$ the Patterson-function calculated on the basis of 429 structure factors derived from the refined structure (statistical disorder model) [26]. This hypo- thetical data set contains 13 symmetry independent rods up to a maximum in-plane momentum transfer $q_{\|}=0.910 \AA^{-1}$, corresponding to the (1 $7 / 20)$ reflection. The structure factor intensities along the rods were calculated in steps of $\Delta l=0.1$ as far as possible and depending on $q_{\|}$assuming $\lambda=1.54 \AA$.

In this case the vectors can be correlated with the maxima of the Patterson-function, although there are still deviations due to truncation errors and the overlap of several similar vectors within the structure.

To summarize we can state that the interpretation of the partial Patterson-function in order to develop a starting model for the structure refinement is severely complicated by its intrinsic properties and due to the low number of reflections that in many cases can be measured in surface $\mathrm{X}$-ray diffraction experiments.

\section{Summary}

Applied to the $\mathrm{Ge}(001)(2 \times 1)$ reconstruction we have presented a depth resolved X-ray superstructure analysis providing information about the rearrangement of the atoms normal to the sample surface. Using two disorder models, a dynamical model with anisotropic thermal parameters, and a statistical model with split positions for the atoms of the first two layers, we confirmed the formation of asymmetric dimers as the main feature of the $(2 \times 1)$ reconstruction. The bond length of the dimer atoms was found to be $R_{\mathrm{D}}=$ $2.44(4) \AA$ (dynamical model) and $K_{\mathrm{D}}=2.46(5) \AA$ (disorder model) whereas the maximum buckling angle was derived to $21^{\circ}$ and $17^{\circ}$, respectively.

Far reaching subsurface layer relaxations were observed already showing up qualitatively by the strong variation of the structure factor intensities versus $q_{z}$. Within the error bars the comparison with the Keating layer relaxation model leads to reasonable agreement. The structure analysis presented in this paper shows the importance to extend the data aquisition to large momentum transfers normal to the sample surface in order to obtain complete insight into the structure. 


\section{Acknowledgements}

The authors would like to thank the members of the workshop staff for their hard trying to keep running the diffractometer as well as the rotating anode. We thank also $\mathrm{C}$. Schamper for his helpful advices how to run the experiments. The assistance of the HASYLAB staff is gratefully acknowledged.

This work is supported by the Bundesminister für Forschung and Technologie under project No. 05 464IAB 8.

\section{References}

[1] J.A. Applebaum and D.R. Hamann, Surf. Sci. 74 (1978) 21.

[2] S.D. Kevan and N.G. Stoffel, Phys. Rev. Lett. 53 (1984) 702.

[3] S.D. Kevan, Phys. Rev. B 32 (1985) 2344.

[4] P. Krüger, A. Mazur, J. Pollmann and G. Wolfgarten, Phys. Rev. B 57 (1986) 1468.

[5] R.J. Hamers, R.M. Tromp and J.E. Demuth, Phys. Rev. B 34 (1986) 5343.

[6] R.J. Culbertson, Y. Kuk and L.C. Feldman, Surf. Sci. 167 (1986) 127.

[7] J.A. Kubby, J.E. Griffith, R.S. Becker and J.S. Vickers, Phys. Rev. B 36 (1987) 6079.

[8] M. Needels, M.C. Payne and J.D. Joannopoulos, Phys. Rev. B 58 (1987) 1765.

[9] R.J. Hamers and U.K. Köhler, J. Vac. Sci. Technol. A 7 (1989) 2854
[10] E. Landemark, R.I.G. Uhrberg, P. Krüger and J. Pollmann, Surf. Sci. Lett. 236 (1990) L359.

[11] M. Mitome and K. Takayanagi, Surf. Sci. 242 (1991) 69.

[12] V.E. Zubkus, P.J. Kundrotas, S.N. Molotkov, V.V. Tatarskij and E.E. Tornau, Surf. Sci. 243 (1991) 295.

[13] J. Dabrovskij and M. Scheffler, App. Surf. Sci., submit* ted.

[14] R.A. Wolkow, Phys. Rev. Lett. 68 (1992) 2636.

[15] P. Eisenberger and W.C. Marra, Phys. Rev. Lett. 46 (1981) 1081.

[16] F. Grey, R.L. Johnson, J.S. Pedersen, R. Feidenhans'1 and M. Nielsen, in: The Structure of Surfaces II, Proc. of the second Int. Conf. on the Structure of Surfaces, Amsterdam (Springer, Berlin, 1988).

[17] For reviews, see: R. Feidenhans'l, Surf. Sci. Rep. 10 (1989) 105;

I.K. Robinson, in: Handbook of Synchrotron Radiation, Eds. D.E. Moncton and G.S. Brown, Vol. 3 (North-Holland, Amsterdam, 1990).

[18] N. Jedrecy, M. Sauvage-Simkin, R. Pinchaux, J. Massies, N. Greiser and V.H. Etgens, Surf. Sci. 230 (1990) 197.

[19] F. Kretschmar, D. Wolf, H. Schulz, H. Huber and H. Plöckl, Z. Krist. 178 (1987) $130^{\circ}$.

[20] R. Rossmann and W. Moritz, to be published.

[21] C. Schamper, H.L. Meyerheim, W. Moritz, and H. Schulz, in: Surface X-Ray and Neutron Scattering, Proc. of the second Int. Conf., Eds. H. Zabel and I.K. Robinson, Bad Honnef, 1991 (Springer, Berlin, 1992).

[22] International Tables for X-Ray Crystallography, Vol. 3 (Kynoch, Birmingham, 1962).

[23] J.S. Pedersen, Surf. Sci. 210 (1989) 238.

[24] R. Rossmann, PhD Thesis, University of Munich, 1992.

[25] M.J. Buerger, Vector space and its application in crystal structure investigation (Wiley, New York, 1959).

[26] It has been checked that there are only small differences between the experimental and the calculated Pattersonfunction of the 80 reflection data set. 